\title{
Cytokinesis failure occurs in Fanconi anemia pathway-deficient murine and human bone marrow hematopoietic cells
}

Patrizia Vinciguerra, ${ }^{1}$ Susana A. Godinho, ${ }^{2}$ Kalindi Parmar, ${ }^{1}$ David Pellman, ${ }^{2}$ and Alan D. D'Andrea

${ }^{1}$ Department of Radiation Oncology, Dana-Farber Cancer Institute, Boston, Massachusetts, USA. ${ }^{2}$ Howard Hughes Medical Institute, Department of Pediatric Oncology, Dana-Farber Cancer Institute, Children's Hospital, Department of Cell Biology, Harvard Medical School, Boston, Massachusetts, USA.

\begin{abstract}
Fanconi anemia (FA) is a genomic instability disorder characterized by bone marrow failure and cancer predisposition. FA is caused by mutations in any one of several genes that encode proteins cooperating in a repair pathway and is required for cellular resistance to DNA crosslinking agents. Recent studies suggest that the FA pathway may also play a role in mitosis, since FANCD2 and FANCI, the 2 key FA proteins, are localized to the extremities of ultrafine DNA bridges (UFBs), which link sister chromatids during cell division. However, whether FA proteins regulate cell division remains unclear. Here we have shown that FA pathway-deficient cells display an increased number of UFBs compared with FA pathway-proficient cells. The UFBs were coated by BLM (the RecQ helicase mutated in Bloom syndrome) in early mitosis. In contrast, the FA protein FANCM was recruited to the UFBs at a later stage. The increased number of bridges in FA pathway-deficient cells correlated with a higher rate of cytokinesis failure resulting in binucleated cells. Binucleated cells were also detectable in primary murine FA pathway-deficient hematopoietic stem cells (HSCs) and bone marrow stromal cells from human patients with FA. Based on these observations, we suggest that cytokinesis failure followed by apoptosis may contribute to bone marrow failure in patients with FA.
\end{abstract}

\section{Introduction}

Fanconi anemia (FA) is a rare inherited disorder characterized by congenital abnormalities, progressive bone marrow failure, and cancer susceptibility. The major cause of death of FA patients is bone marrow failure (1), which usually occurs earlier in life than cancer development. Depletion of bone marrow cells, leading to bone marrow failure, is partly attributed to senescence and/or apoptosis of the HSCs $(2,3)$. Senescence has been observed in bone marrow HSCs from FA mice after exposure to repetitive hyperoxiahypoxia treatments (4). The origin of apoptosis remains unclear and may have diverse underlying causes. Deregulation of apoptotic pathways has been described in FA pathway-deficient bone marrow cells. Accordingly, FA pathway-deficient cells are hypersensitive to the proapoptotic effects of interferon- $\gamma$ and tumor necrosis factor $\alpha$ (5-7). Since FA is a chromosomal instability disorder, another likely cause of apoptosis is the accumulation of increased DNA damage in FA pathway-deficient cells.

FA is genetically heterogeneous, and 13 complementation groups (FANCA, -B, -C, -D1, -D2, -I, -E, -F, -G, -J, -L, -M, and -N) have been identified to date. An additional FA-like disorder has recently been shown to result from biallelic mutation in the gene RAD51C, which suggests that this may represent a 14th FA subtype, FANCO (8). The FA proteins cooperate in a repair mechanism designed to remove DNA crosslinks during the $S$ phase of the cell cycle. The FA pathway is activated when the replication fork encounters a DNA lesion. The first event of the cascade is the activation of a nuclear complex of 8 FA proteins (FANCA, -B, -C, -E, -F, -G, -L, and -M), called the FA core complex (9). Among the FA core complex proteins, only FANCM displays DNA remodeling activity. FANCM contains ATP-dependent translocase

Conflict of interest: The authors have declared that no conflict of interest exists. Citation for this article: J Clin Invest. 2010;120(11):3834-3842. doi:10.1172/JCI43391. activity, which promotes replication fork reversal (10). More recently, FANCM has also been proven to control replication fork progression $(11,12)$. Therefore, besides its role in recruiting the core complex in the chromatin (9), FANCM has been proposed to directly function in DNA replication and repair. The second step of the FA pathway is monoubiquitination of FANCD2 and FANCI by the activated FA core complex (9). Modified FANCD2 and FANCI subsequently accumulate in foci on chromatin, where they colocalize with other repair proteins and participate in DNA interstrand crosslink repair (13). These foci, assembled during $\mathrm{S}$ phase, are believed to disassemble once the damage is repaired, before the exit from $S$ phase.

Recently, 2 studies demonstrated that a small number of FANCD2-I foci persist beyond the $S$ phase of cell cycle and are detected in mitosis $(14,15)$. These persistent foci localize to DNA fragile sites and mark the extremities of ultrafine DNA bridges (UFBs), which link sister chromatids during mitosis (14). Different classes of DNA bridges interlink sister chromatids during cell division. For instance, chromatin bridges - revealed by conventional DNA dyes - contain missegregated DNA and may be derived from dysfunctional telomeres or misregulated chromosome cohesions. The UFBs represent a second class of bridges. They are not detectable by staining with classical DNA dyes and are coated by BLM, the RecQ DNA helicase mutated in Bloom syndrome (BS), and Plk-interacting checkpoint helicase (PICH), an SNF2-family ATPase $(16,17)$. The UFBs, which link the centromeres of sister chromatids, occur naturally in mitosis (17). They represent fully replicated DNA and do not display FANCD2-I foci at their extremities (14). In contrast, the PICH-BLM UFBs, which display FANCD2-I foci at their extremities, are likely to be derived from unresolved replication intermediates. Their generation occurs when replication is incomplete, preferentially at fragile loci under replication stress, but also in between 2 replication forks, leading to the formation of ssDNA catenates between sister chroma- 
A
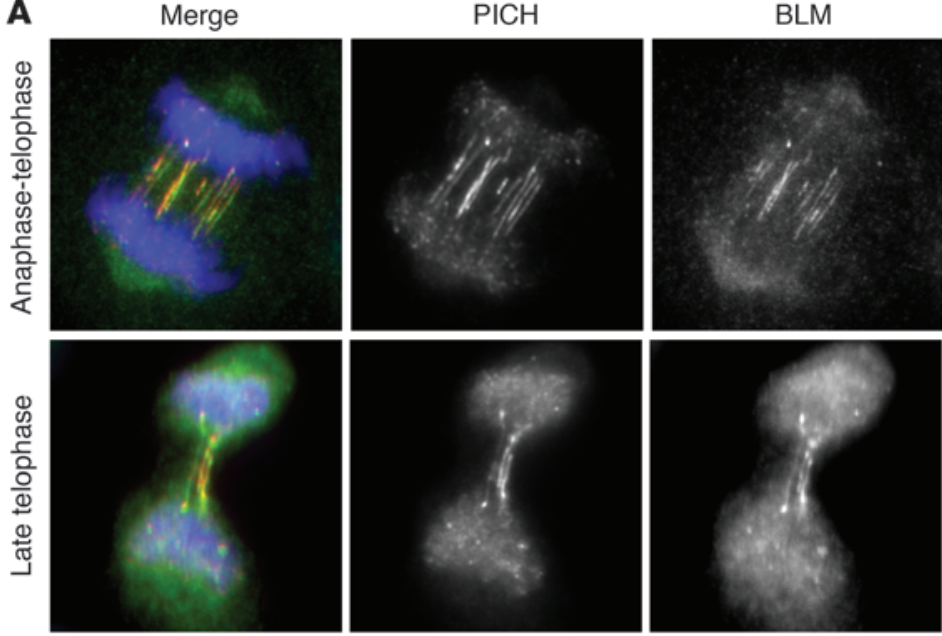

B

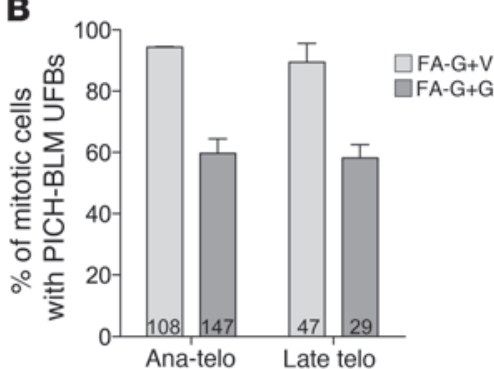

D

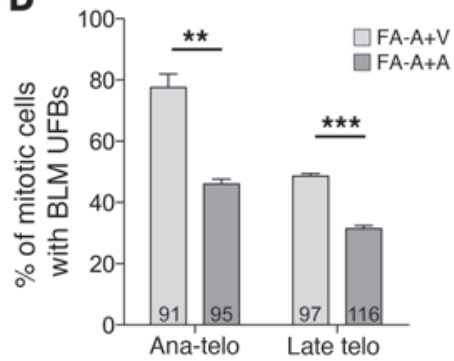

C

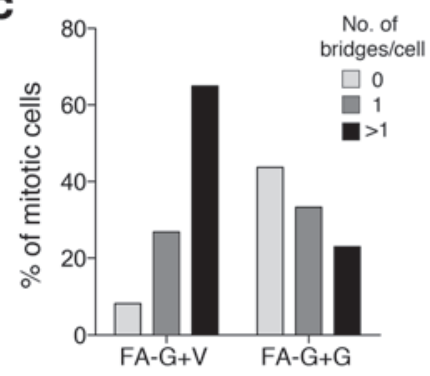

E

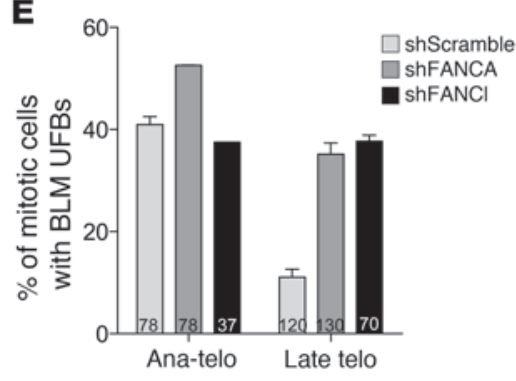

Figure 1

FA pathway-deficient cells exhibit more $\mathrm{PICH}$-BLM bridges in mitosis than do cDNA-complemented or WT cells. (A) FA-G cells transduced with empty vector (FA-G+V) or reconstituted with FANCG cDNA (FA-G+G) were stained for $\mathrm{PICH}$ (red), BLM (green), and DNA (Hoechst; blue). A representative FA-G plus vector mitotic cell with multiple PICH-BLM bridges is shown in anaphase-telophase and in late telophase. Original magnification, $\times 63$. (B) Frequency of mitoses displaying PICH-BLM bridges in FANCG-deficient and -proficient cells. Cells were scored for the presence of PICH-BLM bridges in anaphase-telophase or in late telophase. Data are mean and SD from 2 independent experiments. (C) Distribution of FA-G cells plus vector or FANCG cDNA based on the number of PICH-BLM bridges per mitotic cell. (D) Frequency of mitoses displaying BLM bridges in vector-transduced and reconstituted $F A-A$ cells (FA-A+V and FA-A+A, respectively). Data are mean and SEM from 3 independent experiments. (E) Frequency of mitoses displaying BLM bridges in shScramble, shFANCA, or shFANCl cells. Data are mean and SD from 2 independent experiments. Numbers within bars denote number of mitotic cells analyzed. ${ }^{*} P<0.02$, ${ }^{\star \star \star} P<0.01$, Student's $t$ test. tids. BLM has been shown to participate in the resolution of these bridges and the maintenance of genetic information during mitosis. Indeed, persistent DNA bridges may lead to micronucleation (14).

The role of the FA pathway in the regulation of UFBs during mitosis remained unclear. Here, we showed that FA pathway deficiency affected the presence of UFBs and the completion of cell division. Based on our findings, we propose a mechanism potentially leading to bone marrow failure in patients with FA.

\section{Results}

Increased mitotic DNA bridges in FA pathway-deficient cells. Perturbation of replication by treatment with DNA-damaging agents, such as aphidicolin (APH), mitomycin $\mathrm{C}(\mathrm{MMC})$, and hydroxyurea, has previously been shown to increase the frequency of mitotic cells with UFBs (14). Replication stalling also activates the FA pathway, and FA pathway-deficient cells are hypersensitive to MMC. We therefore investigated the importance of the FA pathway in the formation of PICH-BLM UFBs in untreated populations of cells. We first scored the number of mitotic cells displaying UFBs in FA patient-derived FANCG-deficient fibroblasts (PD326; referred to herein as FA-G cells) and in their FANCG cDNA-corrected counterparts (Figure 1, A and B). The PICH-BLM UFBs were observed in about $90 \%$ of the FA-G cells during anaphase, when the sister chromatids separate, until late telophase, when the chromatin decondenses (Figure 1, A and B). In contrast, only about $50 \%$ of mitotic cells displayed PICH-BLM bridges in the corrected cells (Figure 1B). We also observed an increase in the number of bridges per mitotic cell in uncorrected versus corrected FA-G cells. Indeed, the vast majority of uncorrected cells displayed more than 1 bridge per mitotic cell, whereas the corrected cells usually displayed 0 or 1 bridge (Figure 1C). Consistent with published data (15), we also observed a higher rate of chromosome segregation defects, such as chromatin bridges (i.e., Hoechst positive) and lagging chromosomes or chromosome fragments, in uncorrected versus corrected FA-G cells (Supplemental Figure 1, A and B). The increase in the percentage of mitotic cells displaying PICH-BLM bridges was confirmed in a second FA patient-derived cell line deficient in FANCA (EUFA6914; referred to herein as FA-A cells; Figure 1D). In addition, FA fibroblasts corresponding to FA subtype D1 (i.e., containing biallelic mutations in the downstream 

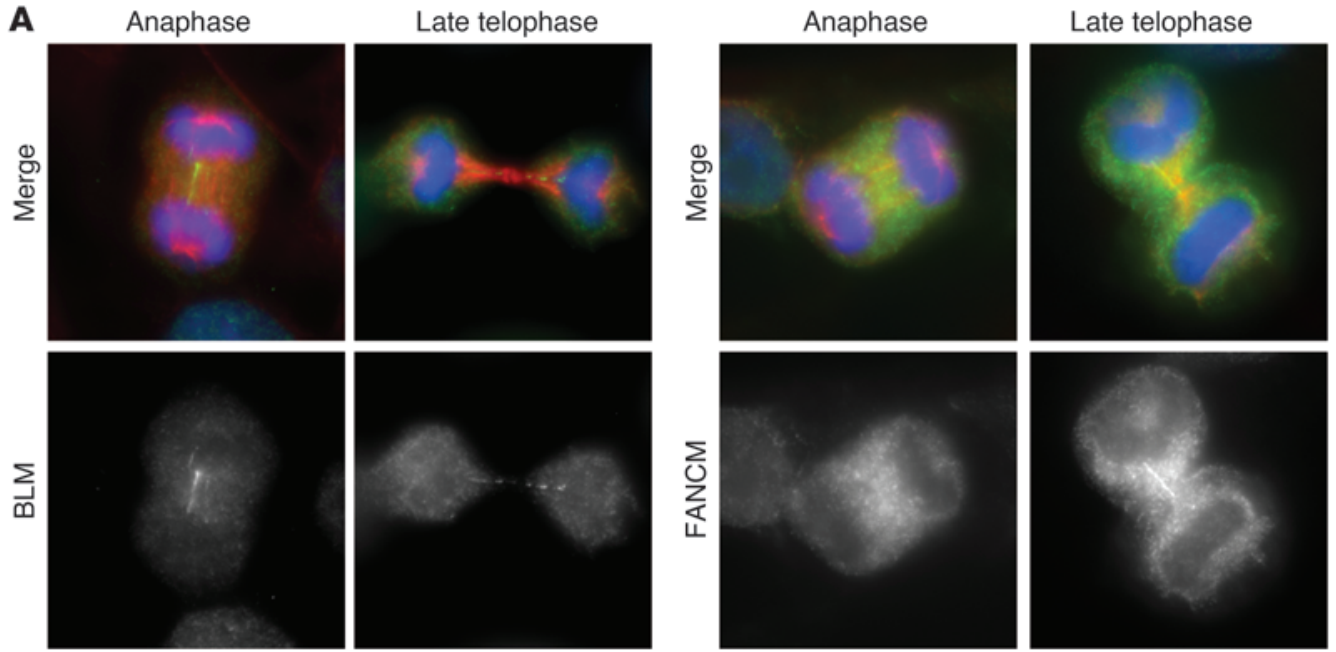

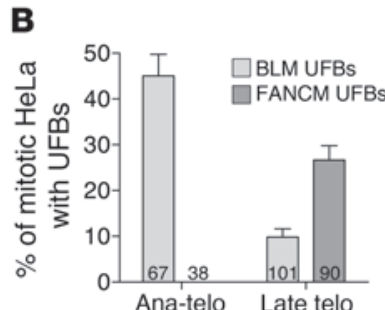

c

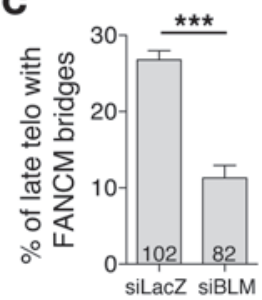

$\mathbf{E}$

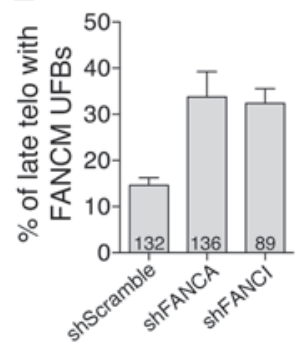

$\mathbf{F}$

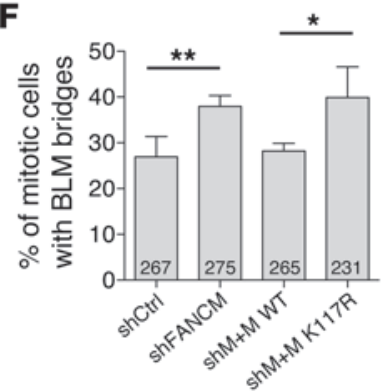

D

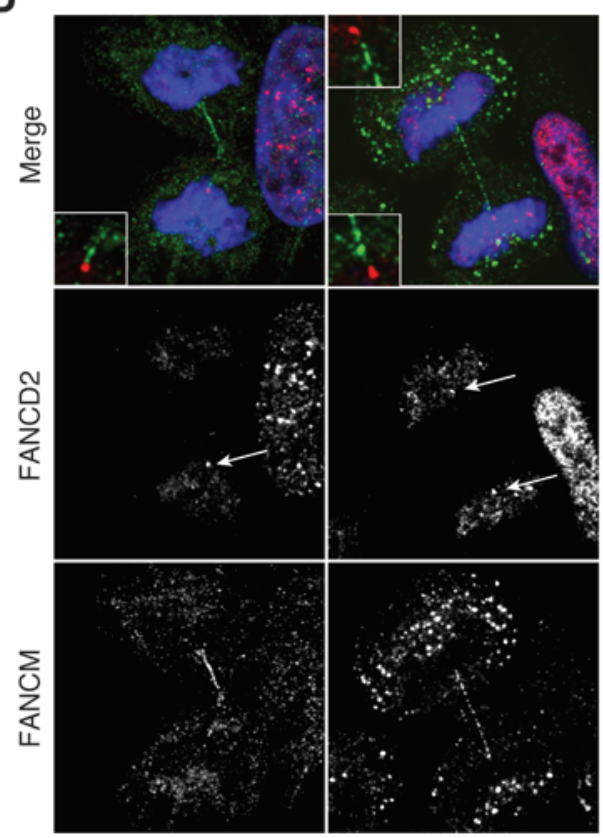

\section{Figure 2}

Localization of FANCM protein to PICH-BLM bridges. (A) Representative images for BLM and FANCM bridges in HeLa cells in anaphase and late telophase. Cells were stained for DNA (blue), microtubules (red), and BLM or FANCM (green). Original magnification, $\times 100$. (B) Frequency of mitotic HeLa cells displaying BLM or FANCM bridges in anaphase and late telophase. Data are mean and SD of 2 independent experiments. (C) Frequency of HeLa cells displaying FANCM bridges in late telophase after treatment with control siRNA (siLacZ) or BLM siRNA for 72 hours. Data are mean and SEM of 3 independent experiments. (D) Representative confocal images of FANCM bridges (green) linking DNA fragile sites marked by FANCD2 foci (red color and arrows). Insets show higher-magnification views of FANCM bridges connecting FANCD2 foci. HeLa cells were treated for 18 hours with aphidicolin $(0.3 \mu \mathrm{M})$ and then released for 6 hours before fixation. Original magnification, $\times 100 ; \times 250$ (insets). (E) Frequency of shScramble, shFANCA, and shFANCI cells displaying FANCM bridges in late telophase. Data are mean and SD from 2 independent experiments. (F) Frequency of control cells (shCtrl), cells stably knocked down for FANCM (shM), or FANCM-deficient cells complemented with WT cDNA (shM+M WT) or expressing FANCMK117R displaying BLM bridges (shM+M K117R) in mitosis. Data are mean and SEM from 3 independent experiments. Numbers within bars denote number of mitotic cells analyzed. ${ }^{\star} P<0.05,{ }^{\star \star} P<0.02$, ${ }^{* \star *} P<0.01$, Student's $t$ test.

BRCA2 gene, which encodes FANCD1) also had increased BLM bridges (Supplemental Figure 1C). Finally, to extend this finding to a different cell type, we examined HeLa cells stably expressing shRNAs targeting FANCA or FANCI (referred to herein as shFANCA and shFANCI, respectively). These cells also exhibited an increase in PICH-BLM UFBs compared with scrambled shRNA control cells (referred to herein as shScramble; Figure 1E), further demonstrating that the FA pathway-deficient cells have an increase in PICH-BLM bridges and missegregated chromatids in mitosis. Functional knockdown of the FA pathway in these transduced HeLa cells was further confirmed by the absence of FANCD2 monoubiquitination (Supplemental Figure 1D). 
A
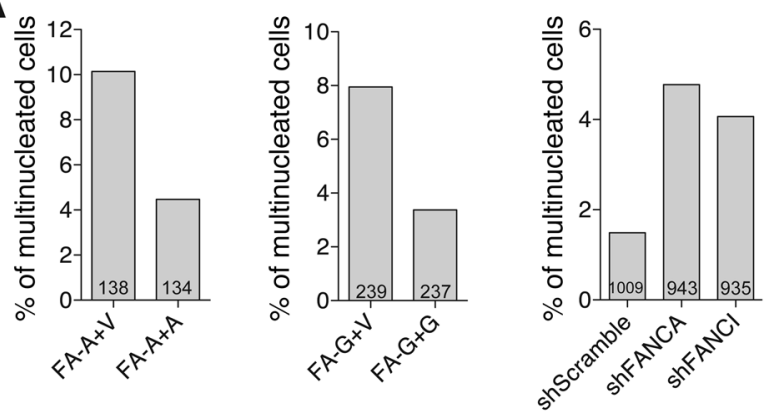

B

C

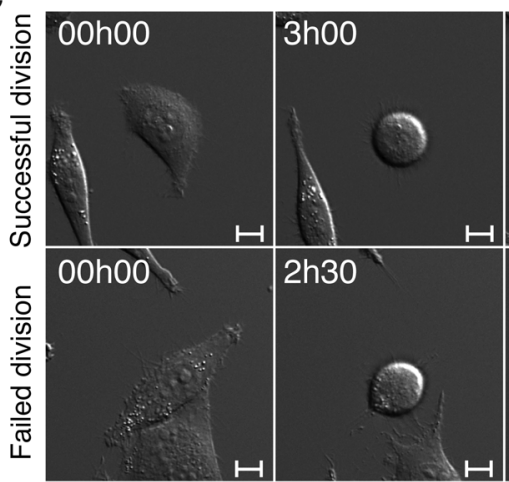

D

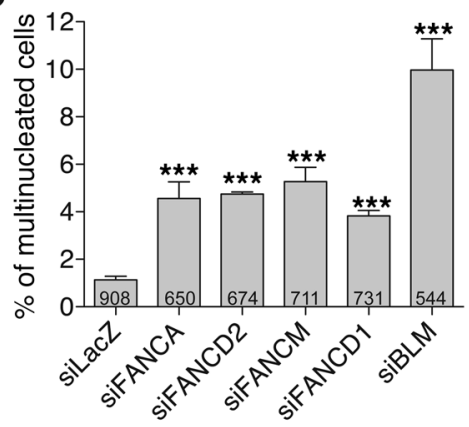

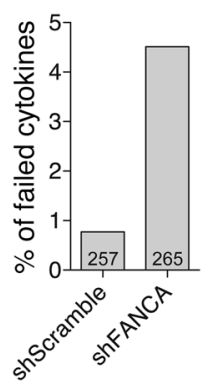

Figure 3

Disruption of the FA pathway results in cytokinesis failure and an increase in multinucleated cells. (A) FA-A and FA-G fibroblasts and corrected counterparts, as well as shScramble, shFANCA, and shFANCl cells, were stained for microtubules and DNA. Mononucleated as well as bi- and multinucleated cells were scored, and the frequency of multinucleated cells (i.e., bi- and multi-) for each type was calculated. (B) Frequency of cytokinesis failure in shScramble and shFANCA cells, followed for 24 hours by live cell imaging. (C) Still frames of representative successful and failed cytokinesis; see Supplemental Movies 1 and 2, respectively. Time is indicated in hours and minutes. Scale bars: $10 \mu \mathrm{M}$. (D) HeLa cells transiently knocked down for a panel of Fanconi genes as well as $B L M$ were analyzed for the presence of bi- and multinucleated cells. Data are mean and SEM from 3 independent experiments (every siRNA was independently compared with siLacZ). (E) Control cells and cells stably knocked down for FANCM, complemented with the WT FANCM CDNA, and expressing FANCMK117R were analyzed for the presence of bi- and multinucleated cells. Numbers within bars denote number of mitotic cells analyzed. ${ }^{* *} P<0.01$, Student's $t$ test.
FANCM localization on UFBs. BLM and the FA core complex proteins are subunits of the same multisubunit complex, named the BRAFT complex (18). Recent evidence supports crosstalk between the FA pathway and the BLM helicase $(14,15,19,20)$. Indeed, FANCM independently recruits both the FA core complex and BLM to the site of the DNA crosslink, promoting combined action of the FA pathway and BLM to repair the damage $(20,21)$. FANCM interacts with BLM via its partner proteins, TopoIII $\alpha$ and RMI1 (20). We therefore hypothesized that FANCM, like BLM, TopoIII $\alpha$, and RMI1 (17), may coat the UFBs.

By immunostaining, we observed FANCM structures similar to the mitotic bridges in late telophase in HeLa cells, which contain an intact FA pathway (Figure 2A). FANCM staining was decreased following siRNA knockdown of FANCM protein, further confirming the specificity of antibody staining (Supplemental Figure 2A). These structures, hereafter referred to as FANCM bridges, were detected in late telophase, when the cleavage furrow has ingressed and chromatin has partially decondensed. In contrast, BLM bridges, which appeared early in anaphase, tended to decrease toward the end of mitosis (Figure 2, A and B). Because of this temporal differ- ence, colocalization of BLM and FANCM was rarely observed (Supplemental Figure 2B). Likewise, PICH, which is known to colocalize with BLM, only rarely colocalized with FANCM (Supplemental Figure 2C). In addition, for rare cells in late telophase, both PICH and FANCM were observed on the same bridges, but in different regions (Supplemental Figure 2D), which suggests that FANCM may replace PICH on the UFBs in late mitosis. We found that BLM depletion reduced FANCM localization to the UFBs (Figure 2C). Given that BLM depletion increases PICH bridges (14), the failure of FANCM to localize to the UFBs cannot be attributable to the decreased number of bridges. Instead, our results suggest that BLM may play a direct role in recruiting FANCM to UFBs late in mitosis, consistent with the published studies describing their direct interaction (20). In addition, we found that, similar to BLM bridges $(14,15)$, FANCM bridges interlinked FANCD2 foci in mitosis after APH treatment (Figure 2D and Supplemental Figure 3). Furthermore, similar to BLM bridges, the number of FANCM bridges increased in FA pathway-deficient cells (Figure 2E). Taken together, our results suggest that FANCM, BLM, and PICH localize to the same UFBs. We hypothesize that FANCM is recruited to 

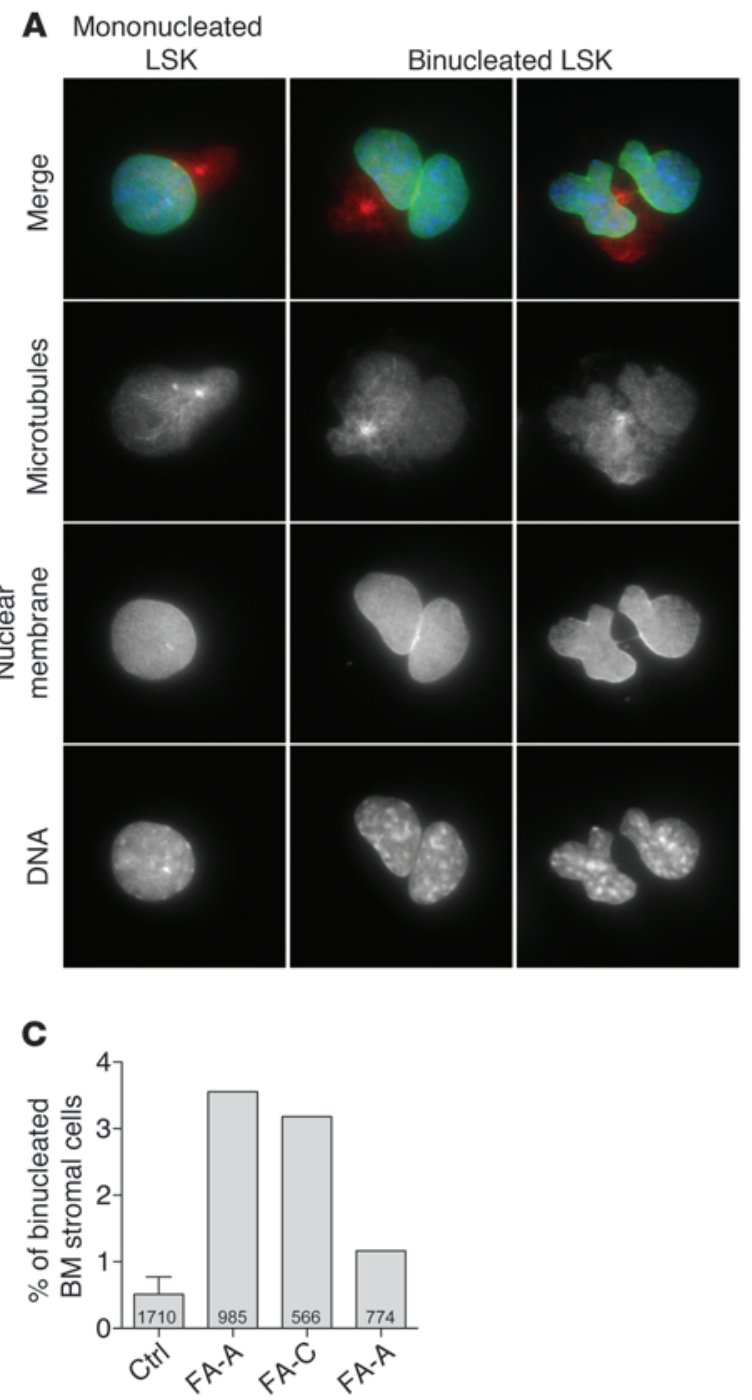

B
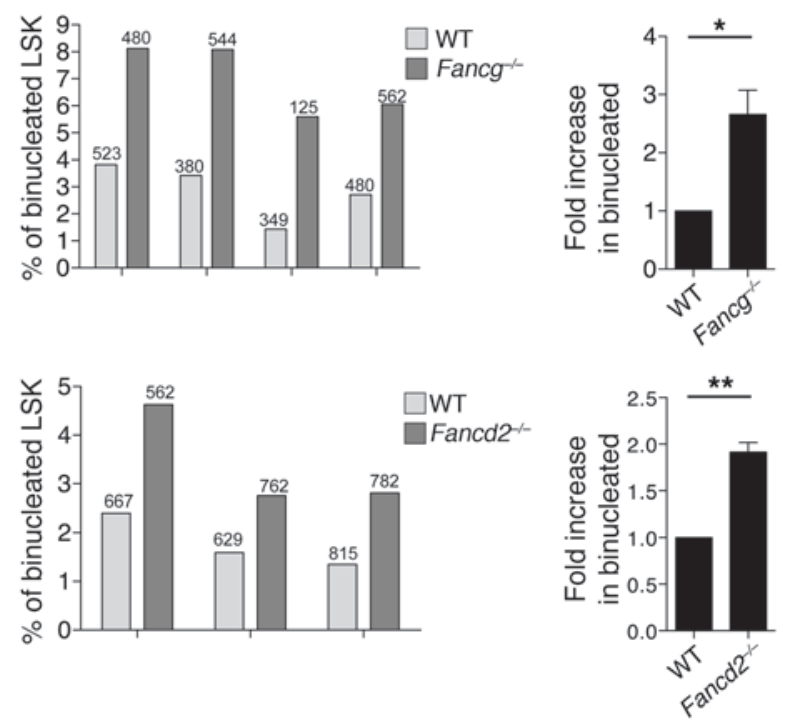

D
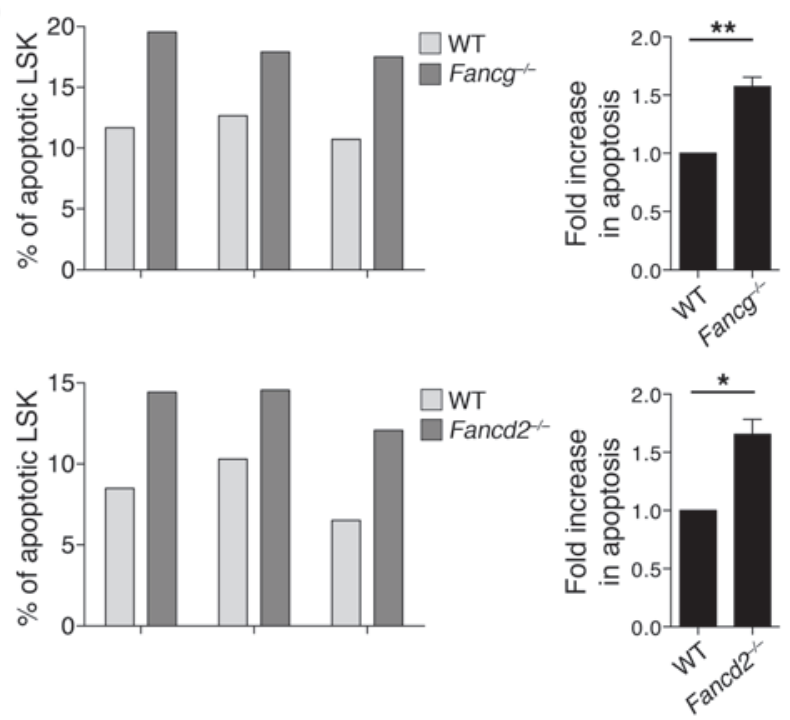

Figure 4

Increased cytokinesis failure in murine Fancg ${ }^{-/}$and Fancd2-/- HSCs and in human FA bone marrow stromal cells. (A) LSK cells from Fancg ${ }^{-/-}$ and Fancd $2^{-1-}$ mice and their respective WT siblings were grown for 48 hours before staining for microtubules (red), nuclear membrane (Lap2; green), and DNA (blue). Representative images of mononucleated as well as binucleated Fancd2-/- LSK cells are shown (note the Lap2 bridge between the 2 nuclei in the third column). Original magnification, $\times 63$. (B) Frequency of binucleated cells in LSK population from bone marrow of 4 pairs of Fancg- ${ }^{--}$and WT siblings and 3 pairs of Fancd2 $2^{--}$and WT siblings. At right, the fold increase in binucleated cells between FA pathway-deficient and WT samples was calculated for each pair of mice; data are mean and SEM. (C) Frequency of binucleated cells in the bone marrow stromal cell population of 3 healthy donors (control, mean and SEM shown) and 3 FA patients. (D) LSK cells from 3 pairs each of Fancd $2^{-/-}$and WT and Fancg ${ }^{-/}$and WT siblings were grown for 48 hours before staining with annexin V. The absolute frequencies of annexin Vpositive apoptotic LSK cells and the fold increase in the number of apoptotic cells is shown. Numbers within and above bars denote number of cells analyzed. ${ }^{* \star} P<0.02,{ }^{*} P<0.05$, Student's $t$ test.

the UFBs later in mitosis, where it replaces BLM and PICH, presumably to facilitate the resolution of persistent UFBs.

The presence of FANCM in the UFBs prompted us to investigate the impact of FANCM deficiency on the PICH-BLM bridges. We used HEK293 cells with stable knockdown of FANCM via expression of an artificial micro RNA sequence specifically targeting FANCM (siRNAmir) (20). Consistent with FANCA and FANCI knockdown (Figure 1E), FANCM deficiency increased the number of BLM bridges compared with control cells (Figure 2F), which suggests that BLM localization to the UFBs does not require FANCM. Complementation of the shFANCM cell line with a siRNA-resistant WT FANCM rescued the defect (Figure 2F).

FANCM plays at least 2 independent functions in the FA pathway: first, it recruits the core complex to chromatin and promotes FANCD2-I ubiquitination; second, it plays a more direct role in repair (22). Accordingly, a patient-derived FANCM-deficient cell 
line is partially deficient for FANCD2 ubiquitination and displays MMC sensitivity (22). Complementation with FANCM WT restores FANCD2 ubiquitination and confers resistance to MMC. Interestingly, expression of an ATPase mutant form of FANCM (FANCMK117R) corrects FANCD2 ubiquitination, but does not restore MMC resistance (22). Therefore, to distinguish between a direct effect of FANCM deficiency on UFBs and an indirect effect through FANCD2 ubiquitination, we quantified the BLM bridges in FANCM siRNAmir cells expressing FANCMK117R (20). The mutant protein did not restore WT levels of BLM bridges (Figure $2 \mathrm{~F}$ ), which suggests that the FANCM ATPase activity plays a direct role in preventing DNA bridges or in their resolution.

Increase cytokinesis failure in FA pathway-deficient cells. Cell division may be inhibited by the presence of chromatin in the cleavage site, possibly resulting in cytokinesis failure and the formation of bi- and multinucleated cells $(23,24)$. We therefore determined whether FA pathway-deficient cells, which exhibit more DNA bridges in mitosis than do FA pathway-proficient cells, are more prone to cytokinesis failure. Indeed, we observed an increase in bi- and multinucleated cells (hereafter grouped and referred to as multinucleated cells) in patient-derived FA-A and FA-G cells versus their corrected counterparts (Figure 3A). As previously described, the nuclei from FA patient-derived cells are often lobated and display irregular shapes that may complicate the differentiation between mononucleated and multinucleated cells (25). Therefore, we determined the percentage of multinucleated cells in shFANCA and shFANCI cells. Similar to the FA patient-derived cell lines, shFANCA and shFANCI cells exhibited an increase in multinucleated cells compared with shScramble cells (Figure 3A).

Binucleated cells can result from cytokinesis failure or from a cell fusion event (24). To distinguish between these possibilities, we followed cell division of shScramble and shFANCA cells by live cell imaging. We confirmed that the binucleated cells scored in these cells lines originated from cytokinesis failure rather than from cell fusion (Figure 3, B and C, and Supplemental Movies 1 and 2). Interestingly, some FA pathway-deficient cells spent more time in cytokinesis than did control cells (Figure 3C). This is consistent with a delay in the completion of cytokinesis caused by the presence of chromatin bridges in the cleavage furrow (26). We also observed apoptosis after cytokinesis failure in FA pathway-deficient cells (Supplemental Figure 4 and Supplemental Movie 3). Finally, acute knockdown of a variety of FA genes caused an increase in multinucleated cells (Figure 3D), demonstrating that cytokinesis failure in FA pathway-deficient cells is not an acquired defect secondary to the long-term depletion of FA proteins. BLM appears to resolve UFBs (27), and BLM deficiency - like FA protein deficiency (Figure 1) - led to an increase in UFBs. In agreement with a model whereby DNA bridges lead to cytokinesis failure, the siRNA against BLM induced an approximately 10 -fold increase in multinucleated cells. In addition, we found that cells expressing FANCM ATPase mutant, which displayed increased UFBs (Figure $2 \mathrm{~F}$ ), also had increased multinucleated cells (Figure 3E).

Increase in multinucleated FA pathway-deficient murine and human HSCs. Multiple studies have examined mouse knockout models for FA (2). Although FA mice do not develop spontaneous bone marrow failure, they do exhibit a defect in HSC proliferation and survival (28-30). We therefore examined the frequency of spontaneous binucleated cells among HSCs from $\mathrm{Fancg}^{-/}$mice, Fancd2 $2^{-/}$mice, and sibling WT controls (Figure 4, A and B). HSCs were isolated as $\mathrm{Lin}^{-} \mathrm{Sca}-1^{+} \mathrm{c}-\mathrm{kit}^{+}(\mathrm{LSK})$ cells from the bone marrow of mice from each representative genotype and were cultured ex vivo for 48 hours to stimulate cell division. Both $\mathrm{Fancg}^{-/}$and Fancd2 $2^{-/-}$HSCs exhibited a higher percentage of binucleated cells than their paired WT sibling controls (Figure 4B). Importantly, when stained for the nuclear membrane component LAP2, some binucleated cells exhibited LAP2 bridges joining the 2 nuclei (Figure 4A, third column). We were also able to detect FANCM bridges surrounded by LAP2 protein in HeLa cells in late mitosis (Supplemental Figure 5). LAP2 accumulates around chromatin when the nuclear membrane reforms at the end of mitosis. Therefore, the presence of LAP2 bridges in binucleated cells is a marker of cytokinesis failure due to chromatin trapped in the cleavage furrow (26). The nuclei of binucleated cells were often very close to each other and may not have allowed detection of a LAP2 bridge (Figure 4A, second column).

Finally, we scored binucleated cells that arise naturally in primary bone marrow stromal cells derived from 3 patients with FA. Although we could not directly investigate HSCs from FA patients, we were able to detect an increase in binucleated cells in primary bone marrow fibroblasts from FA patients (Figure 4C). Collectively, these results suggest that FA pathway-deficient human and murine bone marrow cells exhibit an increase in cytokinesis failure, likely resulting from the presence of unresolved DNA bridges late in mitosis.

The outcome of a successful mitosis is 2 daughter cells with diploid nuclei. Failure to complete cell division results in tetraploid cells, such as the binucleated FA pathway-deficient cells we observed. The fate of most untransformed tetraploid cells is either death by apoptosis or cell cycle arrest and senescence (31-33). Nevertheless, the relative frequency of these 2 fates of tetraploid cells is unknown (34). We used the annexin $V$ assay to detect apoptotic cells among the HSCs from $\mathrm{Fancg}^{-/}$mice, Fancd2 $2^{-/}$mice, and sibling WT controls. HSCs were isolated and cultured as described above for detection of binucleated cells. For some samples, the same population of cells was split and used for either binucleated study or apoptosis measurements. Consistent with a model in which cytokinesis failure may contribute, at least in part, to apoptosis, the increase in binucleated HSCs in Fancd2 $2^{-/-}$and $\mathrm{Fancg}^{-/-}$mice (Figure 4B) correlated with an increase in apoptotic cells (Figure 4D).

\section{Discussion}

The FA pathway participates in maintaining chromosome stability through its role in the DNA damage response and through DNA repair during the $S$ phase of the cell cycle (9). Here we show that the FA pathway is also required for proper cell cycle progression through mitosis and completion of cell division. Cells deficient in FA proteins displayed higher rates of bi- and multinucleated cells as a result of cytokinesis failure. We observed this phenotype in cells deficient in core complex members FANCA, FANCG, and FANCM as well as cells deficient in FANCI and FANCD2, the key effector proteins of the pathway, and in FANCD1, which has a direct role in DNA repair (9). Consistent with the increase in multinucleated cells, we found increased DNA bridges in FA pathway-deficient cells throughout mitosis. DNA bridges have been shown to occlude the division site and are a common cause for cytokinesis failure $(23,24)$. It is therefore possible that the increase in DNA bridges observed in FA pathway-deficient cells leads to a failure in cell division. This finding may also explain the cytokinesis defects observed in FANCD1-deficient cells (ref. 35, Figure 3C, and Supplemental Figure 1C). Our data suggest that a functional FA pathway is required for proper cell division and that mutations in FA genes may result in cytokinesis failure. 
The DNA bridges in FA pathway-deficient cells are either bulky chromatin bridges (Hoechst positive) or PICH-BLM UFBs (Hoechst negative). The increase in chromatin bridges in FA pathway-deficient cells correlates with the previously reported chromosome segregation defects (15). Nevertheless, the origin of these DNA bridges is still unclear; they may result from various defects, including persistent DNA double-strand breaks (36). Interestingly, X-irradiation, a common cause of double-strand breaks, has been reported to induce chromatin bridges and trigger binucleation (37). It is therefore tempting to speculate that unrepaired DNA double-strand breaks resulting from collapsed replication forks may be the source of elevated chromatin bridges in FA pathway-deficient cells.

Our study demonstrates, for the first time to our knowledge, that FA pathway-deficient cells display an increase in PICH-BLM UFBs in mitosis. UFBs represent catenated dsDNA originating from the centromeres of sister chromatids or derived from unresolved replication intermediates originating from regions in which replication is slow, such as fragile site loci $(17,27)$. It is therefore likely that the increase in UFBs in FA pathway-deficient cells is due to the intrinsic DNA repair deficiency of these cells. The mechanisms and pathways involved in the resolution of these bridges are still poorly understood. Also, we showed that FANCM is another protein recruited to the PICH-BLM UFBs. Immunofluorescence staining revealed FANCM localization in the UFBs in late telophase (Figure 2, A and B). In contrast, BLM and PICH were recruited to the UFBs early in mitosis (Figure 2B and ref. 17). FANCM recruitment to the bridges was BLM dependent (Figure 2C), which suggests that FANCM is recruited subsequent to BLM. The UFBs are believed to be resolved in late mitosis, when BLM staining disappears (14). Our findings suggest that the resolution of at least some of the UFBs may proceed beyond BLM disappearance and coincide with FANCM recruitment. FANCM displays additional functions beyond the enhancement of FANCD2-I ubiquitination (22). For instance, FANCM is a potent DNA translocase, which contributes to the replication stress response and controls replication fork progression $(11,12)$. The UFBs linking fragile sites are believed to represent incompletely replicated DNA with ssDNA catenates (14). It is therefore possible that the ATPase PICH and the helicase BLM, together with TopoIII $\alpha$ and RMI1, are involved in a first step of decatenation and/or remodeling and that FANCM is recruited later, during the ssDNA gap-filling step, to control replication progression.

With the exception of rare cell types, such as hepatocytes, which can exist as stable tetraploids (38), tetraploid or polyploid cells are generally genetically unstable and can lead to aneuploidy and ultimately to cancer $(24,39)$. The increase in binucleated cells in FA pathway-deficient cells may represent an early step toward transformation, a characteristic feature of FA, as patients with FA are prone to the development of cancer (40). The organism has developed mechanisms to eliminate tetraploid cells and keep the risk of transformation under control. In normal conditions, most tetraploid cells arrest in a p53-dependent manner, which could lead to senescence or apoptosis $(31,32,41)$. Patients with FA develop bone marrow failure, and apoptosis has been proposed to account, at least in part, for HSC depletion in FA $(3,42)$.

The origin of apoptosis in FA remains unclear and is likely diverse. It may evolve from accumulation of increased DNA damage or from hypersensitivity to apoptotic stimuli. Interestingly, we observed apoptosis following cytokinesis failure in FA pathwaydeficient cells (Supplemental Figure 4 and Supplemental Movie 3). We also detected an increase in apoptosis, which correlated with the increase in binucleated cells in HSCs from FA mice, which suggests that cytokinesis failure may contribute to the depletion of HSCs in FA. It is therefore tempting to propose a model in which an increase in UFBs, as a result of persistent DNA damage, causes an increase in cytokinesis failure and binucleated cells. Binucleated cells may die by apoptosis, leading to the bone marrow failure observed in FA. However, it is noteworthy that patients with other genomic instability syndromes, such as BS, only rarely develop bone marrow disease (43), even though their cells have elevated UFBs and cytokinesis failure. Importantly, p53-mediated apoptosis is attenuated in BS fibroblasts (44), which may explain the absence of bone marrow failure in BS. Mice with FA, which are generally maintained in a protected environment and are not naturally exposed to exogenous challenges such as infections, do not develop spontaneous bone marrow failure either (2). However, treatment of FA mice with the bacterial inflammatory product LPS leads to bone marrow failure (45). LPS mimics infection and elicits a strong immune response, promoting HSC proliferation and differentiation $(46,47)$. Importantly, we observed cytokinesis failure in vitro after inducing proliferation of HSCs. It is therefore likely that cytokinesis failure in proliferating HSCs contributes to the bone marrow failure in FA mice. Taken together, our observations suggest a model in which increased UFBs may lead to the cytokinesis failure observed in FA pathway-deficient HSCs, possibly contributing to bone marrow failure in patients with FA.

\section{Methods}

siRNAs and culture conditions. The synthetic siRNAs used contained the following sequences: siLacZ, 5'-AACGTACGCGGAATACTTCGA-3'; siFANCA, 5'-CAGCGTTGAGATATCAAAGAT-3'; siFANCD2, 5'-TTGGAGGAGATTGATGGTCTA-3'; siFANCM, 5' -AAGCTCATAAAGCTCTCGGAA-3'; siBLM, 5'-TAAGCAGCGATGTGATTTGCA-3'; siFANCD1, 5' -CAGGACACAATTACAACTAAA-3' and 5'-TTGAAGAATGCAGGTTTAATA-3'. siRNA duplexes were transfected using RnaiMax (Invitrogen) following the manufacturer's protocol. 2 rounds of transfections were performed on days 0 and 3 for the experiment in Figure 3C. On day 5, the cells were trypsinized, and part of them was plated on coverslips. Cells on coverslips were fixed on day 6 , and the rest of the cells were collected to assess the level of knockdown by Western blotting.

shRNAs and lentivirus production. The lentiviral vector PLKO.1 shFANCA was purchased from Open Biosystems (TRCN0000118982). The shRNA targeting FANCI (5'-AAGCCAATCCCTAACCTCATC-3') was designed using the siRNA selection program from the Whitehead Institute (48). The lentiviral vector pLKO.1 shScramble was purchased from addgene (plasmid 1864; ref. 49). The pLKO.1 plasmids were transfected in combination with pCMV-dR8.2 dvpr and pCMV-VSVG into 293FT cells using Lipofectamine 2000 (Invitrogen) to produce lentiviral particles. HeLa cells were infected and subsequently grown in puromycin $(1 \mu \mathrm{g} / \mathrm{ml})$ to allow selection. Knockdown efficiency was tested by Western blotting using affinity-purified rabbit sera against FANCA (50) or the N terminus of FANCI (Supplemental Figure 1D).

Mice. Wild-type mice, $\mathrm{Fancg}^{-/-}$and $\mathrm{Fancd}_{2-/-}$ mice, were generated in our laboratory. All the mice experiments were performed in accordance with federal and Institutional Animal Care and Use Committee (IACUC) guidelines. The Animal Care and Use Committee (ACUC) of the Dana-Farber Cancer Institute, Boston, reviewed and approved all the protocols and procedures. Fanc ${ }^{-1-}$ mice were previously described in Yang et al. (51); Fancd2-mice were previously described in Parmar et al. (52). Bone marrow was harvested by flushing tibias and femurs from the hind limbs in HBSS++ (HBSS containing 2\% FBS and 10 mM HEPES buffer; Gibco, Invitrogen). Cells were then passed through 21-gauge needles to obtain single-cell sus- 
pensions. For LSK staining, cells were stained in HBSS++ using biotinylated anti-lineage antibody cocktail (anti-Mac1 $\alpha, \mathrm{Gr}-1, \mathrm{Ter} 119, \mathrm{CD} 3 \mathrm{e}, \mathrm{CD} 4$, CD8a), PE-conjugated anti-Sca-1 antibody (clone e13-161.7), and allophycocyanin-conjugated anti-c-Kit antibody (clone 2B8), followed by staining with a secondary Cychrome-Streptavidin. The samples were analyzed by flow cytometry using a BD FACSAria high-speed sorter. LSK cells were FACS sorted by gating Lin- cells for Sca-1 and c-Kit staining. The isolated LSK cells were cultured in a StemPro serum-free complete medium (Gibco, Invitrogen) containing $40 \mathrm{ng} / \mathrm{ml}$ each murine SCF and murine thrombopoietin and allowed to proliferate for 48 hours. For scoring mono- and binucleated cells, the LSK cells were cytospun on slides and fixed with $4 \%$ paraformaldehyde. For apoptosis detection, cells were stained with FITC-conjugated annexin V and analyzed by FACS. All the antibodies and reagents for apoptosis detection for staining were from BD Biosciences - Pharmingen. For 2 of the 4 pairs of $\mathrm{Fancg}^{-1-}$ and littermate controls used in Figure 4B, the same cells were split and used for both assays.

Human bone marrow stromal cells. Control bone marrow samples were purchased from Lonza (1M-125C). Bone marrow samples from patients with FA were obtained following Dana Farber Cancer Institute Institutional Review Board-approved informed consent. FA patients were diagnosed on clinical grounds with chromosomal breakage test. Primary fibroblast cultures were established from bone marrow aspirates and grown in Chang Medium D (Irvine Scientific). The FA primary cell cultures used in Figure 4C are referred to as DF117, DF1653, and DF1238.

Immunofluorescence and antibodies. For the immunofluorescence experiments, cells were grown on glass coverslips (12-545-83 15CIR-1D; Fisherbrand). In the case of HEK293 cells (Figure $2 \mathrm{~F}$ and Figure 3E) and to allow better adhesion, the coverslips were coated with poly-L-lysine (SigmaAldrich). For quantification of binucleated cells and good preservation of microtubules, cells were fixed with 4\% PFA (catalog no. 15710; Electron Microscopy Sciences) for 15 minutes at room temperature prior to permeabilization ( $0.2 \%$ triton for 5 minutes at room temperature). Fixation and permeabilization were performed simultaneously for UFB detection (i.e., PICH, BLM, and FANCM) as described previously (53). Primary antibodies and dilutions were as follows: mouse anti-FANCD2, 1:80 (catalog no. FI17; Santa Cruz Biotechnology Inc.); rabbit anti-BLM, 1:100 (catalog no. DR1034; Calbiochem); goat anti-BLM, 1:80 (catalog no. C-18; Santa Cruz Biotechnology), used only in Supplemental Figure 2B; rat anti-PICH, 1:600 (gift from E.A. Nigg, University of Basel, Basel, Switzerland); rabbit anti-FANCM, 1:80 (catalog no. sc-98710; Santa Cruz Biotechnology Inc.); mouse anti-Lap2, 1:1,000 (BD Biosciences); mouse anti-tubulin, 1:1,000 (catalog no. DM11A; abcam); rat anti-tubulin, 1:500 (catalog no. YL1/2; Novus Biologicals). All the incubations with primary antibodies were performed for 1 hour at room temperature, followed by 2 washes with PBS. Appropriate secondary antibodies conjugated with Alexa Fluor 488, 568, or 660 were used (1:1,000 dilution; Invitrogen). Incubations with second- ary antibodies were performed in the dark for 50 minutes at room temperature, followed by 2 washes with PBS. Nuclear DNA was detected using Hoechst (1:5,000 dilution, catalog no. 33342; Sigma-Aldrich). Slides were mounted using Prolong Gold antifade reagent from Invitrogen.

Indirect immunofluorescence and live cell imaging. For indirect immunofluorescence, images were acquired with an Axiovert 200M inverted microscope (Zeiss) equipped with a CCD camera (CoolSnap; Photometrics) and Slidebook software (Intelligent Imaging Innovations). Confocal immunofluorescence images (Figure 2D and Supplemental Figure 3) were acquired with a Yokogawa CSU-X1 spinning disk confocal mounted on a Nikon Ti-E inverted microscope (Nikon Instruments) equipped with an Orca ER charge-coupled device (CCD) camera (Hamamatsu Photonics) and Andor iQ software. Images presented in the figures are maximumintensity projections of entire $z$ stacks. For live cell imaging, cells were grown on glass-bottom, 12-well tissue culture dishes (Mattek) and imaged on a TE2000-E2 inverted Nikon microscope equipped with a cooled CCD camera (Orca ER; Hamamatsu Photonics) and the Nikon Perfect Focus system. Differential interference contrast images were captured at multiple points every 4 minutes for 24 hours with a $\times 200.5$ NA Plan Fluor objective. All captured images were analyzed using NIS-Elements software.

Statistics. Statistical analyses were carried out using GraphPad Prism software using a 2-tailed Student's $t$ test. A $P$ value less than 0.05 was considered significant.

\section{Acknowledgments}

We would like to thank Erich A. Nigg for the anti-PICH antibody and Andrew J. Deans and Stephen C. West for the FANCM siRNAmir and FANCMWT or FANCMK117R corrected HEK293 cells. We thank members of the D'Andrea and Pellman laboratories for helpful discussions and Kanchan Mirchandani and Fabio Martinon for critically reading the manuscript. We also thank Lisa Moreau, Kaya Zhu, and Abigail Hamilton for technical help. This work was supported by NIH grants R01DK43889, R01HL52725, and P01CA092584 to A.D. D'Andrea. P. Vinciguerra was supported by a fellowship from the Swiss Foundation for grants in biology and medicine and by Swiss National Science Foundation grant PASMA3-119584. D. Pellman is an Howard Hughes Medical Institute Investigator.

Received for publication April 18, 2010, and accepted in revised form August 18, 2010.

Address correspondence to: Alan D. D'Andrea, Department of Radiation Oncology, Dana-Farber Cancer Institute, Harvard Medical School, 44 Binney Street, Boston, Massachusetts 02115, USA. Phone: 617.632.2112; Fax: 617.632.5757; E-mail: Alan_dandrea@ dfci.harvard.edu.
1. Tischkowitz M, Dokal I. Fanconi anaemia and leukaemia - clinical and molecular aspects. Br J Haematol. 2004;126(2):176-191.

2. Parmar K, D’Andrea A, Niedernhofer LJ. Mouse models of Fanconi anemia. Mutat Res. 2009; 668(1-2):133-140.

3. Du W, Adam Z, Rani R, Zhang X, Pang Q. Oxidative stress in Fanconi anemia hematopoiesis and disease progression. Antioxid Redox Signal. 2008; 10(11):1909-1921.

4. Zhang X, Li J, Sejas DP, Pang Q. Hypoxia-reoxygenation induces premature senescence in FA bone marrow hematopoietic cells. Blood. 2005;106(1):75-85.

5. Whitney MA, et al. Germ cell defects and hematopoietic hypersensitivity to gamma-interferon in mice with a targeted disruption of the Fanconi anemia C gene. Blood. 1996;88(1):49-58.
6. Haneline LS, et al. Multiple inhibitory cytokines induce deregulated progenitor growth and apoptosis in hematopoietic cells from $\mathrm{Fac}^{-/}$mice. Blood. 1998;91(11):4092-4098.

7. Koh PS, Hughes GC, Faulkner GR, Keeble WW, Bagby GC. The Fanconi anemia group $C$ gene product modulates apoptotic responses to tumor necrosis factor-alpha and Fas ligand but does not suppress expression of receptors of the tumor necrosis factor receptor superfamily. Exp Hematol. 1999;27(1):1-8.

8. Vaz F, et al. Mutation of the RAD51C gene in a Fanconi anemia-like disorder. Nat Genet. 2010; 42(5):406-409.

9. Moldovan GL, D'Andrea AD. How the fanconi anemia pathway guards the genome. Annu Rev Genet. 2009;43:223-249.

10. Gari K, Decaillet C, Delannoy M, Wu L, Constanti- nou A. Remodeling of DNA replication structures by the branch point translocase FANCM. Proc Natl Acad Sci U S A. 2008;105(42):16107-16112.

11. Schwab RA, Blackford AN, Niedzwiedz W. ATR activation and replication fork restart are defective in FANCM-deficient cells. EMBO J. 2010; 29(4):806-818.

12. Luke-Glaser S, Luke B, Grossi S, Constantinou A. FANCM regulates DNA chain elongation and is stabilized by S-phase checkpoint signalling. EMBOJ. 2010;29(4):795-805.

13. Knipscheer $P$, et al. The Fanconi anemia pathway promotes replication-dependent DNA interstrand crosslink repair. Science. 2009;326(5960):1698-1701.

14. Chan KL, Palmai-Pallag T, Ying S, Hickson ID. Replication stress induces sister-chromatid bridging at fragile site loci in mitosis. Nat Cell Biol. 2009; 
11(6):753-760

15. Naim V, Rosselli F. The FANC pathway and BLM collaborate during mitosis to prevent micro-nucleation and chromosome abnormalities. Nat Cell Biol. 2009; 11(6):761-768

16. Baumann C, Korner R, Hofmann K, Nigg EA. PICH, a centromere-associated SNF2 family ATPase, is regulated by Plk1 and required for the spindle checkpoint. Cell. 2007;128(1):101-114.

17. Chan KL, North PS, Hickson ID. BLM is required for faithful chromosome segregation and its localization defines a class of ultrafine anaphase bridges. EMBO J. 2007;26(14):3397-3409.

18. Meetei AR, et al. A multiprotein nuclear complex connects Fanconi anemia and Bloom syndrome. Mol Cell Biol. 2003;23(10):3417-3426.

19. Rosado IV, Niedzwiedz W, Alpi AF, Patel KJ. The Walker B motif in avian FANCM is required to limit sister chromatid exchanges but is dispensable for DNA crosslink repair. Nucleic Acids Res. 2009;37(13):4360-4370.

20. Deans AJ, West SC. FANCM connects the genome instability disorders Bloom's Syndrome and Fanconi Anemia. Mol Cell. 2009;36(6):943-953.

21. Vinciguerra P, D'Andrea A. FANCM: A landing pad for the Fanconi Anemia and Bloom's Syndrome complexes. Mol Cell. 2009;36(6):916-917.

22. Singh TR, et al. Impaired FANCD2 monoubiquitination and hypersensitivity to camptothecin uniquely characterize Fanconi anemia complementation group M. Blood. 2009;114(1):174-180.

23. Mullins JM, Biesele JJ. Terminal phase of cytokinesis in D-98s cells. J Cell Biol. 1977;73(3):672-684.

24. Ganem NJ, Storchova Z, Pellman D. Tetraploidy, aneuploidy and cancer. Curr Opin Genet Dev. 2007; 17(2):157-162.

25. Willingale-Theune J, Schweiger M, Hirsch-Kauffmann M, Meek AE, Paulin-Levasseur M, Traub P. Ultrastructure of Fanconi anemia fibroblasts. J Cell Sci. 1989;93(pt 4):651-665.

26. Steigemann P, et al. Aurora B-mediated abscission checkpoint protects against tetraploidization. Cell. 2009;136(3):473-484.

27. Chan KL, Hickson ID. On the origins of ultra-fine anaphase bridges. Cell Cycle. 2009;8(19):3065-3066.

28. Navarro S, et al. Hematopoietic dysfunction in a mouse model for Fanconi anemia group D1. Mol Ther. 2006;14(4):525-535.

29. Haneline LS, et al. Loss of FancC function results in decreased hematopoietic stem cell repopulating ability. Blood. 1999;94(1):1-8.

30. Carreau M, Gan OI, Liu L, Doedens M, Dick JE, Buchwald M. Hematopoietic compartment of Fanconi anemia group $\mathrm{C}$ null mice contains fewer lineage-negative $\mathrm{CD} 34+$ primitive hematopoietic cells and shows reduced reconstruction ability. Exp Hematol. 1999;27(11):1667-1674.

31. King RW. When $2+2=5$ : the origins and fates of aneuploid and tetraploid cells. Biochim Biophys Acta. 2008;1786(1):4-14

32. Ganem NJ, Pellman D. Limiting the proliferation of polyploid cells. Cell. 2007;131(3):437-440.

33. Castedo $M$, et al. Apoptosis regulation in tetraploid cancer cells. EMBO J. 2006;25(11):2584-2595.

34. Glotzer M. The molecular requirements for cytokinesis. Science. 2005;307(5716):1735-1739.

35. Daniels MJ, Wang Y, Lee M, Venkitaraman AR Abnormal cytokinesis in cells deficient in the breast cancer susceptibility protein BRCA2. Science. 2004; 306(5697):876-879.

36. Acilan C, Potter DM, Saunders WS. DNA repair pathways involved in anaphase bridge formation. Genes Chromosomes Cancer. 2007;46(6):522-531.

37. Huang $\mathrm{H}$, et al. Abnormal cytokinesis after X-irradiation in tumor cells that override the G2 DNA damage checkpoint. Cancer Res. 2008;68(10):3724-3732.

38. Guidotti JE, Bregerie O, Robert A, Debey P, Brechot C, Desdouets C. Liver cell polyploidization: a pivotal role for binuclear hepatocytes. J Biol Chem. 2003;278(21):19095-19101.

39. Fujiwara T, Bandi M, Nitta M, Ivanova EV, Bronson RT, Pellman D. Cytokinesis failure generating tetraploids promotes tumorigenesis in p53-null cells. Nature. 2005;437(7061):1043-1047.

40. Rosenberg PS, Greene MH, Alter BP. Cancer incidence in persons with Fanconi anemia. Blood. 2003;101(3):822-826.

41. Andreassen PR, Lohez OD, Lacroix FB, Margolis
RL. Tetraploid state induces p53-dependent arrest of nontransformed mammalian cells in G1. Mol Biol Cell. 2001;12(5):1315-1328.

42. Ridet A, et al. Deregulated apoptosis is a hallmark of the Fanconi anemia syndrome. Cancer Res. 1997;57(9):1722-1730.

43. German J. Bloom's syndrome. Dermatol Clin. 1995; 13(1):7-18

44. Wang XW, et al. Functional interaction of p53 and BLM DNA helicase in apoptosis. J Biol Chem. 2001;276(35):32948-32955.

45. Sejas DP, et al. Inflammatory reactive oxygen species-mediated hemopoietic suppression in Fanccdeficient mice. J Immunol. 2007;178(8):5277-5287.

46. Scumpia PO, et al. Cutting edge: bacterial infection induces hematopoietic stem and progenitor cell expansion in the absence of TLR signaling. J Immunol. 2010;184(5):2247-2251.

47. Zhang P, Nelson S, Bagby GJ, Siggins R 2nd, Shellito JE, Welsh DA. The lineage-c-Kit+Sca-1+ cell response to Escherichia coli bacteremia in Balb/c mice. Stem Cells. 2008;26(7):1778-1786.

48. Yuan B, Latek R, Hossbach M, Tuschl T, Lewitter F. siRNA Selection Server: an automated siRNA oligonucleotide prediction server. Nucleic Acids Res. 2004;32(Web Server issue):W130-W134.

49. Sarbassov DD, Guertin DA, Ali SM, Sabatini DM. Phosphorylation and regulation of Akt/ PKB by the rictor-mTOR complex. Science. 2005; 307(5712):1098-1101

50. Kupfer GM, Naf D, Suliman A, Pulsipher M, D'Andrea AD. The Fanconi anaemia proteins, FAA and FAC, interact to form a nuclear complex. Nat Genet. 1997;17(4):487-490.

51. Yang Y, et al. Targeted disruption of the murine Fanconi anemia gene, Fancg/Xrcc9. Blood. 2001; 98(12):3435-3440

52. Parmar K, et al. Hematopoietic stem cell defects in mice with deficiency of Fancd2 or Usp1. Stem Cells. 2010;28(7):1186-1195.

53. Wang LH, Schwarzbraun T, Speicher MR, Nigg EA. Persistence of DNA threads in human anaphase cells suggests late completion of sister chromatid decatenation. Chromosoma. 2008;117(2):123-135. 